\title{
Electrochemically tunable ultrafast optical response of graphene oxide
}

Ulaş Kürüm, Okan Öner Ekiz, H. Gul Yaglioglu, Ayhan Elmali, Mustafa Ürel, Hasan Güner, Alpay Koray Mızrak, Bülend Ortaç, and Aykutlu Dâna

Citation: Appl. Phys. Lett. 98, 141103 (2011);

View online: https://doi.org/10.1063/1.3573797

View Table of Contents: http://aip.scitation.org/toc/apl/98/14

Published by the American Institute of Physics

\section{Articles you may be interested in}

Ultrafast carrier dynamics and saturable absorption of solution-processable few-layered graphene oxide Applied Physics Letters 98, 121905 (2011); 10.1063/1.3570640

Ultrafast relaxation dynamics of hot optical phonons in graphene Applied Physics Letters 96, 081917 (2010); 10.1063/1.3291615

Measurement of ultrafast carrier dynamics in epitaxial graphene Applied Physics Letters 92, 042116 (2008); 10.1063/1.2837539

Temperature tunability of photonic crystal fiber filled with $\mathrm{Fe}_{3} \mathrm{O}_{4}$ nanoparticle fluid Applied Physics Letters 98, 021103 (2011); 10.1063/1.3540647

Femtosecond pump-probe studies of reduced graphene oxide thin films Applied Physics Letters 96, 173106 (2010); 10.1063/1.3421541

Photoluminescence and band gap modulation in graphene oxide Applied Physics Letters 94, 111909 (2009); 10.1063/1.3098358

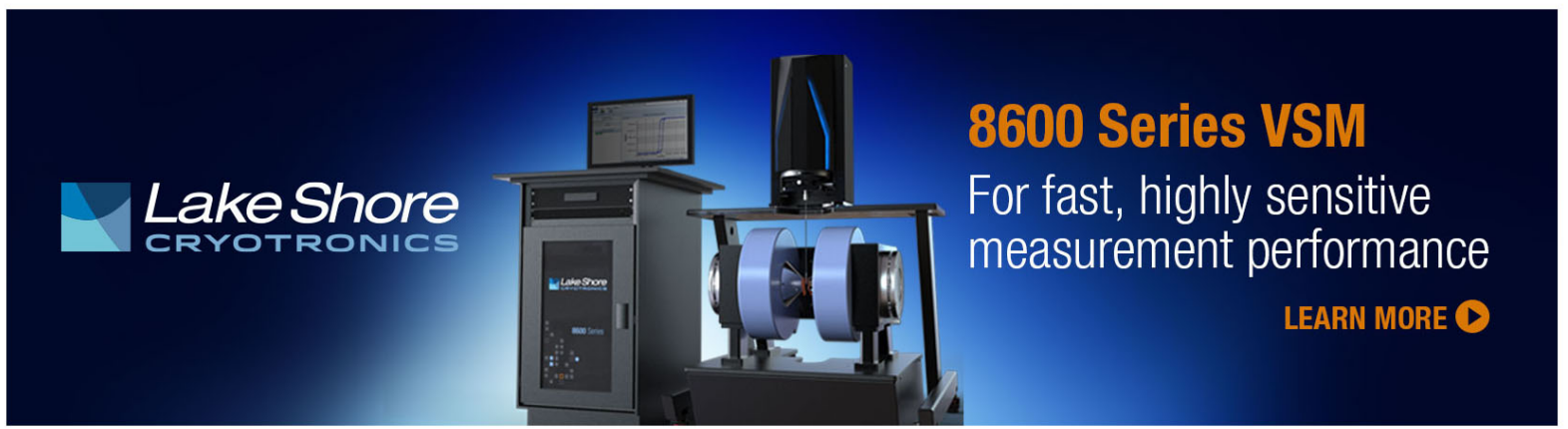




\title{
Electrochemically tunable ultrafast optical response of graphene oxide
}

\author{
Ulaş Kürüm, ${ }^{1}$ Okan Öner Ekiz, ${ }^{2}$ H. Gul Yaglioglu, ${ }^{1, a)}$ Ayhan Elmali, ${ }^{1}$ Mustafa Ürel, ${ }^{2}$ \\ Hasan Güner, ${ }^{2}$ Alpay Koray Mızrak, ${ }^{2}$ Bülend Ortaç, ${ }^{2}$ and Aykutlu Dâna ${ }^{2, a)}$ \\ ${ }^{1}$ Department of Engineering Physics, Faculty of Engineering, Ankara University, 06100 Ankara, Turkey \\ ${ }^{2}$ UNAM Institute of Materials Science and Nanotechnology, Bilkent University, Bilkent 06800 \\ Ankara Turkey
}

(Received 12 December 2010; accepted 6 March 2011; published online 5 April 2011)

\begin{abstract}
We demonstrate reversible and irreversible changes in the ultrafast optical response of multilayer graphene oxide thin films upon electrical and optical stimulus. The reversible effects are due to electrochemical modification of graphene oxide, which allows tuning of the optical response by externally applied bias. Increasing the degree of reduction in graphene oxide causes excited state absorption to gradually switch to saturable absorption for shorter probe wavelengths. Spectral and temporal properties as well as the sign of the ultrafast response can be tuned either by changing the applied bias or exposing to high intensity femtosecond pulses. (C) 2011 American Institute of Physics. [doi:10.1063/1.3573797]
\end{abstract}

Ultrafast dynamics and nonlinear optical response of graphene has been the subject of considerable research. ${ }^{1,2}$ Graphene is known to exhibit wideband nonlinear saturable absorption (SA). It is accepted that due to Pauli blocking, nonlinear absorption (NA) in graphene is not allowed. Graphene oxide (GO) is an insulator, with an effective energy gap that depends on the stoichiometry. ${ }^{3,4}$ In GO, it was found that two-photon absorption dominate the NA for picosecond pulses, whereas for nanosecond pulses excited state absorption also influences the nonlinear response. ${ }^{5}$ It has been previously observed that, GO can be reduced controllably by annealing at below $300 \mathrm{C}$ (low-T) in ambient atmosphere. ${ }^{6,7}$ Interruption of the annealing results in partially reduced GO (PRGO). GO has been reduced by exposing to a photographic camera flash ${ }^{8}$ or femtosecond laser. ${ }^{9}$ Very recently, we studied reversible electrical reduction and oxidation of GO. ${ }^{10}$ Nanoscale inspection showed that GO islands segregate within graphene, and a two-dimensional heterostructure nanomesh forms during electrochemical oxi- dation. Here, we study the effect of the oxidation level on nonlinear optical properties of GO. We demonstrate that both electrochemically induced reversible reduction and optically induced photoreduction in GO result in changes in the nonlinear optical properties of GO thin films. We present the carrier dynamics and nonlinear optical properties of such films, studied by ultrafast wavelength-dependent pumpprobe spectroscopy. We show that ultrafast response of GO can be tuned by both reduction procedure.

The preparation, characterization, linear optical, and electrochromism properties of GO were very recently reported..$^{10}$ We study the electrical reduction in GO in air, using multilayer GO thin films deposited on metalized glass substrates. The two terminal devices consist of thin $(\sim 10-50 \mathrm{~nm}) \mathrm{Pd} / \mathrm{Au}$ planar contacts, separated by $0.3-0.6$ $\mathrm{mm}$, with a thin multilayer GO film covering both contacts and in between [Fig. 1(a)]. The degree of chemical reduction and linear absorption spectrum can be tuned by applying a (a)
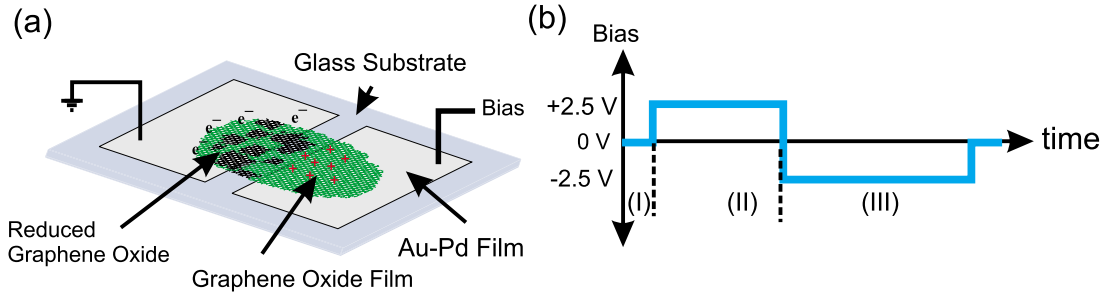

(d)

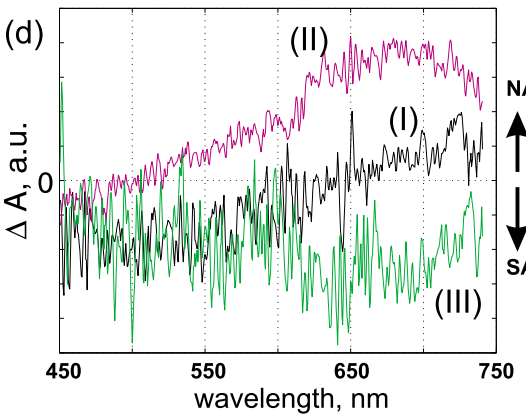

$\Delta \mathrm{A}$

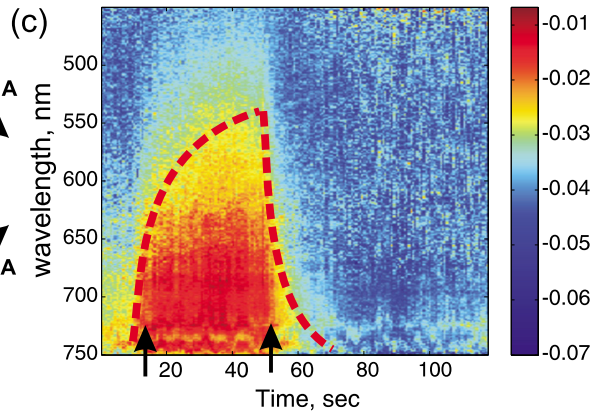

FIG. 1. (Color online) (a) Schematic description of the device used in the measurements. (b) The applied bias voltage profile and (c) normalized differential probe transmission $(\Delta \mathrm{A}$ as defined in text) are plotted as a function of wavelength and time. Wavelength dependent switching between saturable and NA is observed upon application of the bias (pump excitation energy of $0.5 \mu \mathrm{J}$, center wavelength of $790 \mathrm{~nm}$, pulse width of 44 fs). The dashed lines are guidelines for the eyes, delineating the transition wavelength between saturable and NA. (d) Differential absorption $(\Delta \mathrm{A})$ plotted as a function of wavelength for three representative points with different bias conditions [regions I, II, and III in (c)] showing NA and SA.

${ }^{\text {a)} E l e c t r o n i c ~ a d d r e s s e s: ~ g u l . y a g l i o g l u @ e n g . a n k a r a . e d u . t r ~ a n d ~ a y k u t l u @ u n a m . b i l k e n t . e d u . t r . ~}$ 

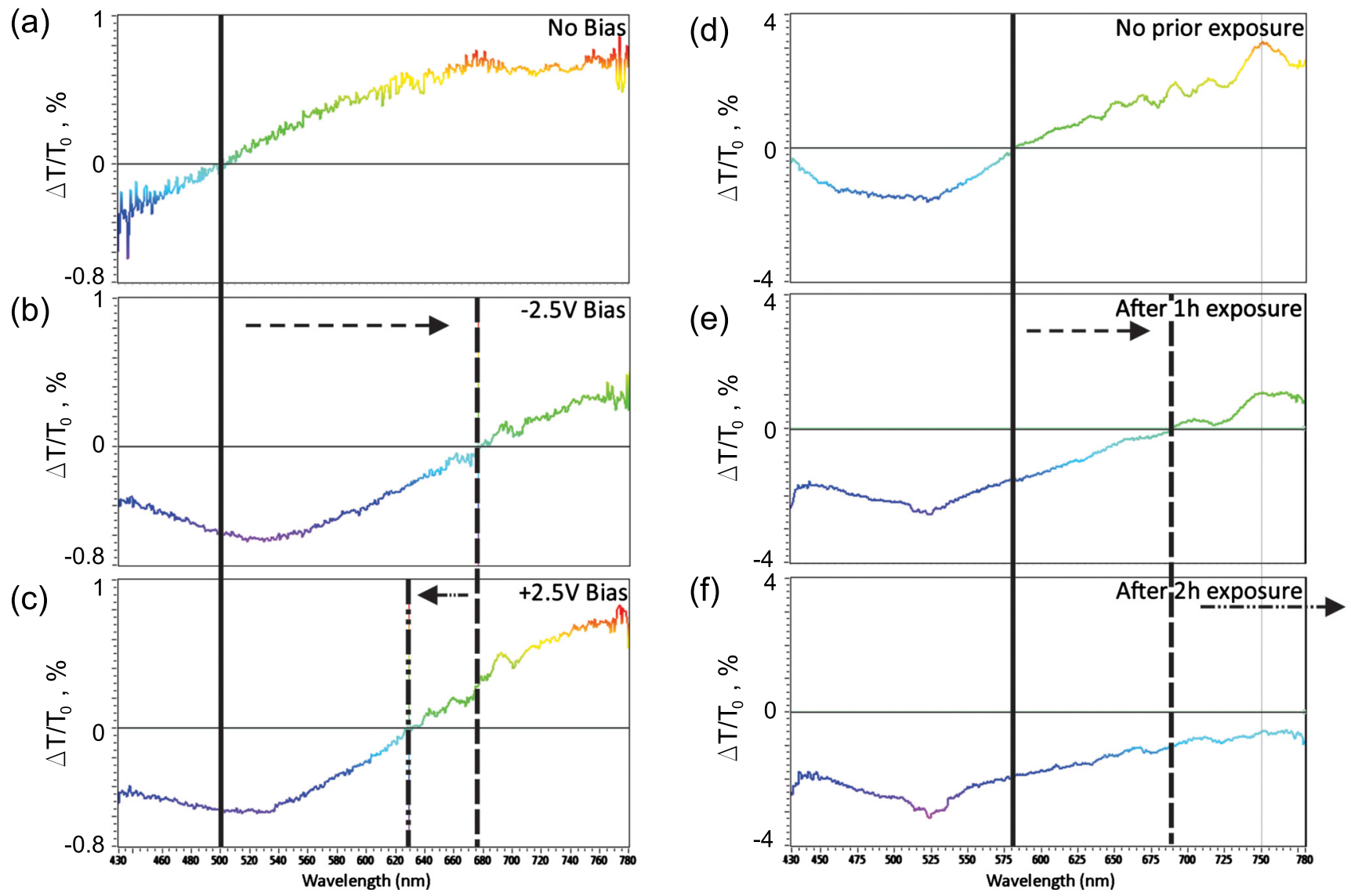

FIG. 2. (Color online) Differential absorption of probe at zero pump-probe delay. (a)-(c) show voltage bias dependence. (d)-(f) Changes in the response upon high-intensity pump exposure for various durations. Similar changes are observed for electrochemical and photothermal reduction.

fixed voltage difference to the contacts. A gradual increase in the absorption edge as a function of applied bias is observed. ${ }^{10}$ During the cyclic voltage sweeps, it is seen that the linear optical transparency of the films change between partially opaque and transparent states. Electrochemically induced oxidation/reduction takes place within a time scale on the order of seconds for applied bias voltages of $\pm 2.5 \mathrm{~V}$. We infer the thickness of the films to be 30-60 nm using optical measurements, assuming the films have graphenelike optical absorption during the reduced state.

Wavelength-dependent pump probe measurements were performed by using Ti:sapphire laser amplifier-optical parametric amplifier system (Spectra Physics, Spitfire Pro XP, TOPAS) with 44 fs pulse duration and $1 \mathrm{KHz}$ repetition rate. Commercial pump probe experimental setup with white light continuum probe beam (Spectra Physics, Helios) was used. Experiments were performed with 400, 590, and $790 \mathrm{~nm}$ pump wavelengths. Pulse duration inside pump-probe experimental set up was measured via cross correlation of pump and probe pulses and it was found to be around $100 \mathrm{fs}$. Positive sign of pump probe data $\left(\Delta \mathrm{A}=\Delta \mathrm{T} / \mathrm{T}_{0}\right)$ corresponds to NA whereas negative sign of $\Delta \mathrm{A}$ corresponds to $\mathrm{SA}$ in our convention.

We investigate the ultrafast optical response of the films while the voltage bias is applied. A film left in the oxidized state after multiple redox cycles is characterized by the pump-probe technique. Figure 1 shows normalized change in the absorption of the white light continuum spectra, measured using $790 \mathrm{~nm}, 0.5 \mu \mathrm{J}$ pump energy with a typical pulse width of $100 \mathrm{fs}$, upon application of a time varying voltage [profile shown in Fig. 1(b)]. Although initially SA is dominant over the whole spectrum, NA appears rapidly (in a matter of seconds) upon oxidation [Figs. 1(c) and 1(d)]. We attribute this to an increase in the degree of oxidation of GO, causing SA to gradually switch to NA for short probe wavelengths, possibly due to modification of the band-structure of the material. The oxidation state can be controlled electrochemically and GO can be reduced by application of a negative bias [Fig. 1(c)]. Even when electrochemically or thermooptically induced partial reduction takes place, we still observe NA at longer probe wavelengths $(\sim 750 \mathrm{~nm})$, suggesting only a partial reduction in GO. Application of a -2.5 $\mathrm{V}$ bias causes the advancing of SA behavior toward longer wavelengths, suggesting the presence of a stoichiometrydependent effective band-gap. The ultrafast response can therefore be modified upon application of the bias. The experiments were performed with different pump wavelengths, and similar results with varying decay times and modulation depths are observed.

Figures 2(a)-2(c) show differential absorption of probe $(430-780 \mathrm{~nm})$ with simultaneously applied pump $(\sim 0.5 \mu \mathrm{J}$, $400 \mathrm{~nm}, 44 \mathrm{fs}$ ) for GO; without applying bias, applying -2.5 $\mathrm{V}$ for $5 \mathrm{~s}$ and applying $+2.5 \mathrm{~V}$ for $5 \mathrm{~s}$, respectively. It is clearly seen that the ultrafast optical absorption of the samples can be switched from SA to NA reversibly depending on the wavelength and degree of the reduction. We also performed similar experiments using femtosecond pulses $(\sim 5 \mu \mathrm{J}, 400 \mathrm{~nm}, 44 \mathrm{fs})$ without applying bias. Figures 2(d)-2(f) show differential absorption of probe (430-780 $\mathrm{nm}$ ) with simultaneously applied pump for $\mathrm{GO}$, without prior 
(a)

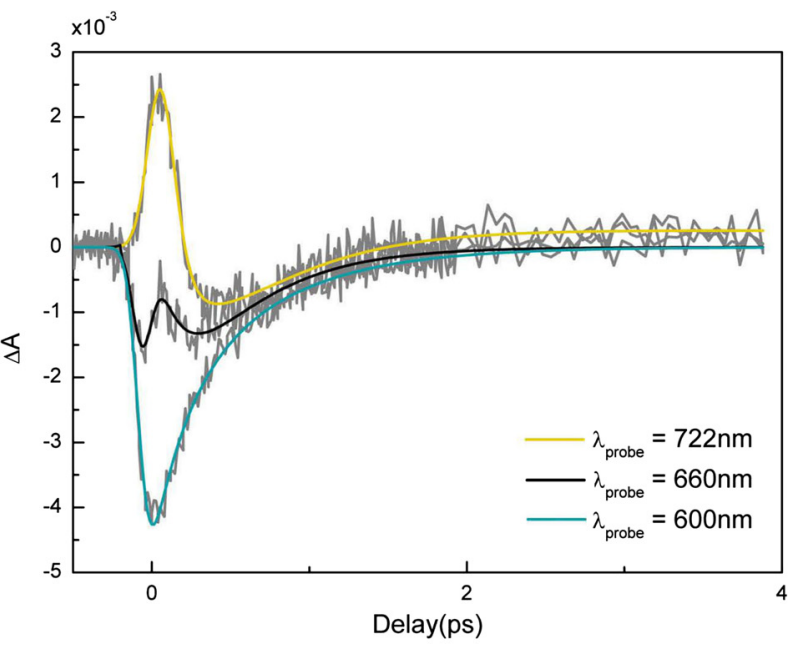

(b)

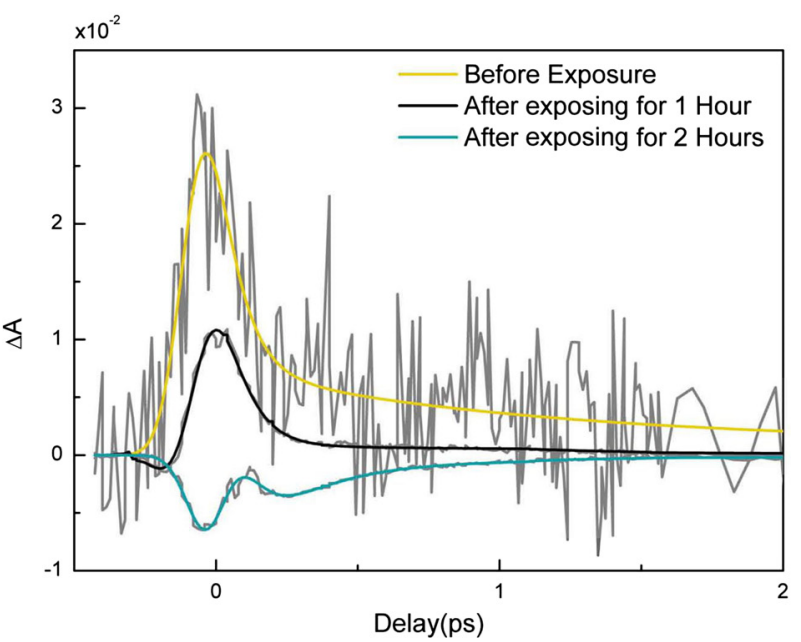

FIG. 3. (Color online) (a) Temporal behavior of the saturated and NA responses at different probe wavelengths after applying bias voltage. (b) Temporal behavior of the saturated and NA responses at $750 \mathrm{~nm}$ probe wavelength depending on the exposure time to pump (pump excitation energy of $5 \mu \mathrm{J}$, center wavelength of $790 \mathrm{~nm}$, pulse width of $44 \mathrm{fs}$ ).

exposure, after exposing to pump for $1 \mathrm{~h}$ and for $2 \mathrm{~h}$, respectively. Photoreduction in GO takes places by exposing high intensity femtosecond pulses and this process is irreversible. Applying high intensity femtosecond pulses and applying negative bias cause similar effects on the differential absorption characteristics of GO.

At intermediate oxidation stoichiometries achieved by application of a $-2.5 \mathrm{~V}$ bias or by exposing to high intensity femtosecond pulses, if the probe wavelength is chosen near the SA/NA transition, NA signal starts appearing within the SA signal region [Figs. 3(a) and 3(b)]. For example, as seen in Fig. 3(a), the NA peak appears within 100-200 fs of the arrival of the pump pulse. Such a time scale is between the carrier-carrier scattering and carrier-phonon scattering and the NA process disappears in a very short time, leaving the tail of carrier-phonon scattering visible. In a simplistic view, it can be assumed that the simultaneous appearance of SA and NA is the result of isolated regions of the material acting as graphene and GO. The optical response is averaged over the $300 \mu \mathrm{m}$ diameter illumination spotsize and the material behaves like an effective material with multiple components. In such a case, the persistence of NA for a variety of pump wavelengths even when the pump photon energy is less than the apparent absorption edge of GO, requires that multiphoton absorption or absorption of the probe through intermediate states must be present. Previous reports on observations of simultaneous presence of SA and NA in graphene offered an explanation in terms of doping of the graphene layers. Switching as a function of the probe wavelength from saturation to NA was observed around $1.78 \mu \mathrm{m}$ for doped epitaxial graphene on $\mathrm{SiC} .{ }^{11}$ The NA of the probe seen at longer wavelengths and saturation seen at shorter wavelengths were attributed to the shifting in the Fermi level due to doping. As molecules bind to the surface of the graphene, depending on the molecule and the binding location graphene experiences a charge transfer as a donor and acceptor, thus changing the Fermi level, carrier density, and electrical resistance of graphene. ${ }^{12}$ In addition, the band structure itself may be modified depending on the type, location, and density of the binding species. ${ }^{4}$ In our case, the observed NA/saturation behavior may be attributed to such modifications of Fermi level or band structure upon electrochemical stimulus. The zero crossing wavelengths can be tuned from $550 \mathrm{~nm}$ to beyond $800 \mathrm{~nm}$ (our probe range). Although the exact origin of the electrochromic effects in the ultrafast regime is disputable, due to the SA/NA cancellation effect, for carefully chosen pump-probe wavelength and degree of reduction combinations, the nonlinear response of graphene can be tuned to display a very fast $(<100 \mathrm{fs})$ effective NA decay time constant.

In conclusion, the linear and nonlinear optical properties are highly dependent on the electronic structure of the layers and the degree of reduction, thereby on bias and/or the intensity of the femtosecond pulses. The nonlinear optical characteristics of GO can be switched from SA to excited state absorption depending on the wavelength and the degree of reduction. Photothermal and electrochemical reduction are observed to produce changes with similar characteristics in the ultrafast response. Electrochemical tunability of the ultrafast response opens up the possibility of using GO-PRGO for tunable ultrafast optical device applications.

${ }^{1}$ J. M. Dawlaty, S. Shivaraman, M. Chandrashekhar, F. Rana, and M. G. Spencer, Appl. Phys. Lett. 92, 042116 (2008).

${ }^{2}$ G. Xing, H. Guo, X. Zhang, T. C. Sum, and C. H. H. A. Huan, Opt. Express 18, 4564 (2010).

${ }^{3}$ J.-A. Yan, L. Xian, and M. Y. Chou, Phys. Rev. Lett. 103, 086802 (2009).

${ }^{4}$ D. W. Boukhvalov and M. I. Katsnelson, J. Am. Chem. Soc. 130, 10697 (2008).

${ }^{5}$ Z. Liu, Y. Wang, X. Zhang, Y. Xu, Y. Chen, and J. Tian, Appl. Phys. Lett. 94, 021902 (2009).

${ }^{6}$ I. Jung, D. A. Dikin, R. D. Piner, and R. S. Ruoff, Nano Lett. 8, 4283 (2008).

${ }^{7}$ V. López, R. S. Sundaram, C. Gomez-Navarro, D. Olea, M. Burghard, J. Gomez-Herrero, F. Zamora, and K. Kern, Adv. Mater. (Weinheim, Ger.) 21, 1 (2009).

${ }^{8}$ L. J. Cote, R. Cruz-Silva, and J. Huang, J. Am. Chem. Soc. 131, 11027 (2009).

${ }^{9}$ Y. Zhang, L. Guo, S. Wei, Y. He, H. Xia, Q. Chen, H. Sun, and F. Xiao, Nanotoday 5, 15 (2010).

${ }^{10}$ O. Ö. Ekiz, M. Ürel, H. Güner, A. K. Mizrak, and A. Dâna "Reversible electrical reduction and oxidation of graphene oxide," ACS Nano (in press).

${ }^{11}$ D. Sun, Z. Wu, C. Divin, X. Li, C. Berger, W. A. de Heer, P. N. First, and T. B. Norris, Phys. Rev. Lett. 101, 157402 (2008).

${ }^{12}$ Y. Zhu, S. Murali, W. Cai, X. Li, W. Ji, J. R. Potts, and R. S. Ruoff, Adv. Mater. (Weinheim, Ger.) 22, 3906 (2010). 